\title{
Targeting endothelin receptors $A$ and $B$ attenuates the inflammatory response and improves locomotor function following spinal cord injury in mice
}

\author{
JIAN GUO $^{1}$, YIQIAO LI ${ }^{2}$, ZHENNIAN HE ${ }^{1}$, BIN ZHANG ${ }^{1}$, YONGHUAN LI ${ }^{1}$, JIANGHUA HU ${ }^{1}$, \\ MINGYUAN HAN ${ }^{1}$, YUANLIN XU ${ }^{1}$, YONGFU LI ${ }^{1}, \mathrm{JIE} \mathrm{GU}^{1}$, BO DAI ${ }^{1}$ and $\mathrm{ZHONG} \mathrm{CHEN}^{3}$ \\ ${ }^{1}$ Department of Orthopaedic Surgery, ${ }^{2}$ Central Laboratory, Ningbo Beilun People Hospital, Ningbo, Zhejiang 315800; \\ ${ }^{3}$ Department of Orthopaedic Surgery, The First Affiliated Hospital of College of Medicine, \\ Zhejiang University, Hangzhou, Zhejiang 310000, P.R. China
}

Received November 21, 2013; Accepted April 1, 2014

DOI: 10.3892/ijmm.2014.1751

\begin{abstract}
After spinal cord injury (SCI), the disruption of blood-spinal cord barrier by activation of the endothelin (ET) system is a critical event leading to leukocyte infiltration, inflammatory response and oxidative stress, contributing to neurological disability. In the present study, we showed that blockade of ET receptor A (ETAR) and/or ET receptor B (ETBR) prevented early inflammatory responses directly via the inhibition of neutrophil and monocyte diapedesis and inflammatory mediator production following traumatic SCI in mice. Long-term neurological improvement, based on a series of tests of locomotor performance, occurred only in the spinal cord-injured mice following blockade of ETAR and ETBR. We also examined the post-traumatic changes of the microenvironment within the injured spinal cord of mice following blockade of ET receptors. Oxidative stress reflects an imbalance between malondialdehyde and superoxide dismutase in spinal cord-injured mice treated with vehicle, whereas blockade of ETAR and ETBR reversed the oxidation state imbalance. In addition, hemeoxygenase-1, a protective protease involved in early SCI, was increased in spinal cord-injured mice following the blockade of ETAR and ETBR, or only ETBR. Matrix metalloproteinase-9, a tissue-destructive protease involved in early damage, was decreased in the injured spinal cord of mice following blockade of ETAR, ETBR or a combination thereof. The findings of the present study therefore suggested an association between ETAR and ETBR in regulating early
\end{abstract}

Correspondence to: Dr Zhong Chen, Department of Orthopaedic Surgery, The First Affiliated Hospital of College of Medicine, Zhejiang University, 79 Qingchun Road, Hangzhou, Zhejiang 310000, P.R. China

E-mail: zhongchenmed@163.com

Key words: endothelin receptors, inflammatory response, long-term neurological improvement, oxidative stress pathogenesis of SCI and determining the outcomes of long-term neurological recovery.

\section{Introduction}

The blood-spinal cord barrier (BSCB) is a highly specialized structural, transport and biochemical barrier within the central nervous system (CNS). Similar to the blood-brain barrier (BBB), the BSCB is primarily formed by endothelial cells interconnected by tight junctions, which limits passive diffusion of blood-borne solutes and actively transports nutrients into the spinal cord $(1,2)$. BSCB dysfunction leading to early inflammatory response and oxidative stress contributes to secondary pathogenesis following traumatic spinal cord injury (SCI) (3-10). BSCB disruption by traumatic SCI also generates harmful substances, including endothelins (ETs) (11-15), matrix metalloproteinases (MMPs) $(7,9,10)$, inflammatory cytokines and reactive oxygen species (ROS) $(3-6,8)$ that can induce programmed neuronal death and permanently impair neuron function. This is exemplified by studies showing that the blockade of ET receptors or ET-converting enzyme (ECE) activity in brain endothelial cells and glia, which results in endothelial hyperpermeability and cerebral vasoconstriction, reduces leukocyte infiltration into the injured spinal cord, which is associated with significant recovery of motor and neurological functions following SCI (16-18). Therefore, the brain ET system is considered to be a therapeutic target of SCI.

The ET system consists of two G-protein-coupled receptors (ET receptors A and B, ETRs), three peptides (ET-1, ET-2 and ET-3), and two activating peptidases (ECE-1 and ECE-2). It is the most potent vasoconstrictor and is essential for embryonic development, vascular remodeling, and wound healing $(19,20)$. Excessive activation of the ET system can be detrimental, leading to multidimensional pathological conditions, including $\mathrm{BBB}$ or BSCB disruption following ischemic brain injury and traumatic SCI, as well as inflammation $(20,21)$. For example, the ET system is found throughout the brain as its components are synthesized in vascular, neuronal, and glial cells. Expression pattern of ET system components in many discrete 
brain areas suggests a variety of possible functions (19-22). ET-1 is the predominant neural ET and plays a critical role in abnormal vascular endothelial cell permeability and inflammation after SCI, while the upregulation of ET-1 modulates behavior and the metabolism without affecting cerebral blood flow (23). In addition, ETs exert their effects through the activation of ET receptor A (ETAR) and/or ET receptor B (ETBR) $(19,22,23)$. In normal spinal cord, ETAR is found predominantly in vascular smooth muscle cells and primary afferent nerve fibers, whereas ETBR is abundantly expressed in endothelial cells, radial glia, a small population of astrocytes, and epithelial tissues $(23,24)$. Following SCI, vascular ETAR/ETBR activation plays a critical role in post-traumatic ischemia, and astrocyte-only ETBR activation is associated with reactive gliosis (23-25). However, until recently, there was a lack of consensus regarding which ETR subtype was the key determinant of oxidative stress and functional recovery after SCI, and there has been controversy regarding the exact cellular targets of ETAR and ETBR in the injured spinal cord.

In the present study, we examined the effects of ETAR and/or ETBR blockade on early SCI pathogenesis and long-term neurological recovery in murine models. Our results demonstrated that ETR blockage markedly reduced inflammatory responses and oxidative stress, ameliorated MMP-9 activation, and enhanced long-term neurological function in SCI mice. The results confirm additive pathogenic roles for ETAR and ETBR in the injured spinal cord and may aid in the identification of a set of putative therapeutic targets for neural tissue damage after SCI.

\section{Materials and methods}

SCI. All the procedures were conducted in accordance with the National Institutes of Health Guide for the Care and Use of Laboratory Animals and with approval from the Animal Subjects Committee at Zhejiang University. Adult female C57BL/6 (18-22 g) mice were anesthetized with chloral hydrate $(500 \mathrm{mg} / \mathrm{kg}$ ) and subjected to a moderate spinal cord contusion injury. A laminectomy was performed at the T9 level, and a 2 -g weight was dropped $5 \mathrm{~cm}$ onto the exposed dura mater. After SCI, the skin was closed with wound clips. Animal body temperature was maintained at $37^{\circ} \mathrm{C}$ with a warming blanket throughout the surgery and during the recovery from anesthesia (26). For the sham-operated controls (SHAM), the animals underwent a T9 laminectomy without contusion injury.

Drug treatment. BQ123 and/or BQ788 (both from Sigma, St. Louis, MO, USA) dissolved in sterile phosphate-buffered saline (PBS) were administered to corresponding SHAM and SCI mice (SHAM + BQ123, SHAM + BQ788, SHAM + BQ123 + BQ788, SCI + BQ123, SCI + BQ788 and $\mathrm{SCI}+\mathrm{BQ} 123+\mathrm{BQ788})$ via intraperitoneal injection $(10 \mathrm{mg} / \mathrm{kg}$, respectively) (27-29) at 1 day before SCI and then further treated once a day for 6 weeks for behavioral testing or for the indicated time-points (2, 4, 6 and $24 \mathrm{~h}$ and 4 days) for other experiments post-injury. PBS for vehicle control (VEH) was administered in corresponding SHAM and SCI mice (SHAM + VEH and SCI + VEH). Significant side effects resulting from BQ123 and/or BQ788 treatment, such as changes in body weight or an increase in mortality, were not observed during our experiments.
Leukocyte infiltration assessment. To confirm the depletion of neutrophils, three blood smears, prepared 1 day post-injury were processed with a Hema $3{ }^{\circledR}$ stain kit (Fisher Scientific, Pittsburgh, PA, USA). At least 300 white blood cells were counted, and the percentage of neutrophils relative to the total number of white blood cells was determined. To confirm monocyte depletion, blood samples were taken at 4 days post-injury. A hematology automated white blood cell analyzer (HemaVet $^{\circledR} 850$ ) was used to quantify monocytes. Data are presented as relative percentages relative to the total number of white blood cells.

Quantitative polymerase chain reaction ( $q P C R)$. Total RNA was prepared with the RNeasy kit (Qiagen). Complementary DNA synthesis and reverse transcriptase PCR were performed using a previously described method (30). The primers used were: tumor necrosis factor- $\alpha$ (TNF- $\alpha$ ), forward, 5'-CCCAGA CCCTCACACTCAGAT-3' and reverse, 5'-TTGTCCCTT GAGAGAACCTG-3'; interleukin-1 $\beta$ (IL-1 $\beta$ ), forward, 5'-GCA GCTACCTATGTCTTGCCCGTG-3' and reverse, 5'-GTCGTT GCTTGTCTCTCCTTGTA-3'; IL-6, forward, 5'-AAGTTT CTCTCCGCAAGATACTTCCAGCCA-3' and reverse, 5'-AGGCAAATTTCCTGGTTATATCCAGTT-3'; inducible nitric oxide synthase (iNOS), forward, 5'-CTCCATGACTCT CAGCACAGAG-3' and reverse, 5'-GCACCGAAGATATCC TCATGAT-3'.

Enzyme-linked immunosorbent assay (ELISA). To determine cytokine levels, the lesion site was rapidly dissected and homogenized in PBS at $24 \mathrm{~h}$ after SCI. After centrifugation at $4^{\circ} \mathrm{C}$ for $15 \mathrm{~min}$ at $900 \mathrm{x} \mathrm{g}$, the supernatants were used to measure the concentrations of TNF- $\alpha$, IL- $1 \beta$ and IL-6 (R\&D Systems, Minneapolis, MN, USA), iNOS, malondialdehyde (MDA), and superoxide dismutase (SOD) (Cusabio Biotech Co., Ltd., Hubei, China) using corresponding ELISA kits.

Western blot analysis. A 0.5-cm length of cord, centered over the site of impact and representing the epicenter, was lysed on ice for $30 \mathrm{~min}$ with $50 \mathrm{mM}$ Tris- $\mathrm{HCl}$ (pH 7.5), $150 \mathrm{mM} \mathrm{NaCl}$, $1 \mathrm{mM}$ EDTA, $1 \mathrm{mM}$ EGTA, $25 \mathrm{mM} \mathrm{NaF}, 5 \mathrm{mM}$ sodium pyrophosphate, $1 \mathrm{mM} \mathrm{Na}_{3} \mathrm{VO}_{4}$, and protease inhibitors (Roche). Cell lysates were clarified by centrifugation at $12,000 \mathrm{x} \mathrm{g}$ for $15 \mathrm{~min}$ at $4^{\circ} \mathrm{C}$, and the supernatants were collected and assayed for the protein concentration using a BCA protein assay kit (Pierce, Rockford, IL, USA). Total cell lysates were prepared, and western blot analysis was performed as described previously (30). The antibodies used were: anti-hemeoxygenase-1 (anti-HO-1, sc-10789; Santa Cruz Biotechnology, Inc., Santa Cruz, CA, USA), anti-MMP-9 (ab7299; Abcam), anti$\alpha / \beta$-tubulin (no. 2148; Cell Signaling Technology). Tubulin served as a loading control.

Gelatin zymography. MMP-9 activity at 24-h post-injury was examined by gelatin zymography based on a previously described method (26). The epicenter of the injured spinal cord $(0.5 \mathrm{~cm}$ in length) was homogenized in lysis buffer containing $50 \mathrm{mM}$ Tris- $\mathrm{HCl}(\mathrm{pH} \mathrm{8.0)}, 150 \mathrm{mM} \mathrm{NaCl}$, $1 \%$ NP-40, $0.5 \%$ deoxycholate, and $0.1 \%$ SDS. Then, $50-\mu \mathrm{g}$ protein samples were loaded onto $8 \%$ SDS-polyacrylamide gels and copolymerized with gelatin $(1 \mathrm{mg} / \mathrm{ml}$; Sigma). After 

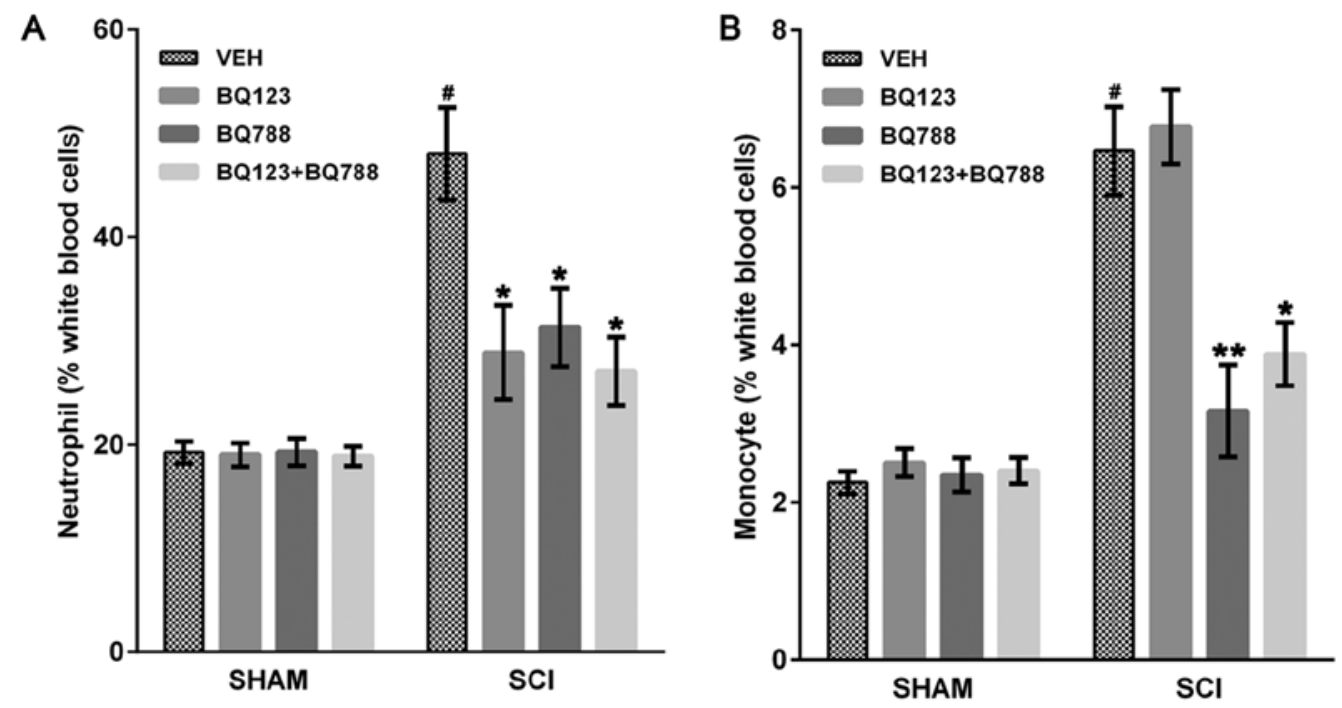

Figure 1. Treatment with BQ123 and/or BQ788 reduces neutrophils and monocytes, respectively, in the blood of spinal cord injury (SCI) mice. Data are the mean \pm standard error of the mean (SEM) of three independent experiments. ${ }^{*} \mathrm{P}<0.05,{ }^{* *} \mathrm{P}<0.01, \mathrm{SCI}$ mice treated with BQ123 and/or BQ788 vs. SCI + vehicle control (VEH) mice; " $\mathrm{P}<0.01$, SCI + VEH mice vs. SHAM mice (n=3/group). (A) The number of neutrophils in blood was manually counted in blood smears stained with the Hema $3^{\circledR}$ Stain kit. (B) The number of monocytes in blood was quantified using an automated white blood cell analyzer (HemaVet).

electrophoresis, renaturation was achieved by incubation of the gel in 2.5\% Triton X-100 for $30 \mathrm{~min}$ and in substrate buffer $\left(50 \mathrm{mM}\right.$ Tris- $\mathrm{HCl}$ at $\mathrm{pH} 8.5,5 \mathrm{mM} \mathrm{CaCl}_{2}$ ) for $48 \mathrm{~h}$ at $37^{\circ} \mathrm{C}$. The gel was stained with Coomassie blue solution for $4 \mathrm{~h}$ and then de-stained with $40 \%$ methanol $/ 10 \%$ acetic acid. For quantitative analysis, gels were scanned, and the positive band was measured using NIH ImageJ software.

Behavioral analysis. Three different behavioral tests were performed to evaluate functional improvements after SCI. The 9-point Basso Mouse Scale (BMS) was used to examine locomotor recovery in an open field $\left(53 \times 10^{8} \times 5.5 \mathrm{~cm}\right)(31)$. This rating scale takes into account limb movement, stepping, coordination, and trunk stability. Mice were tested at 1 and 3 days and weekly thereafter until euthanasia at 6 weeks post-injury. Performance on a rotarod and the ability to traverse a wire grid were evaluated, in sequence, at 40,41 and 42 days post-injury. Three experiments were conducted daily, with a total of nine trials for each test. In each of these tests, the average score from each mouse was used to calculate the mean.

Statistical analysis. Statistical analysis was performed using GraphPad Prism (GraphPad Software, Inc., La Jolla, CA, USA). Statistical significance was defined at $\mathrm{P}<0.05$. Data were presented as the mean \pm standard error of the mean (SEM) of three independent experiments.

\section{Results}

Blockade of ETAR and ETBR reduced leukocyte infiltration in SCI mice. As previously described (32), the early leukocyte influx contributes to secondary pathogenesis in the injured spinal cord. Therefore, we investigated the effects of ETR blockade on leukocyte influx by performing differential blood cell counts. Compared with each SHAM mouse, SCI + VEH mice showed an elevated number of neutrophils and monocytes (Fig. 1). The number of neutrophils in peripheral blood was reduced in SCI mice at $24 \mathrm{~h}$ after blockade of ETAR, ETBR or both (Fig. 1A). Blockade of ETBR or ETAR and ETBR reduced circulating monocytes 4 days after SCI (Fig. 1B). Notably, blockade of only ETAR did not affect the number of circulating monocytes in SCI mice (Fig. 1B).

Blockade of ETAR and ETBR inhibited inflammatory mediator expression in SCI mice. After SCI, leukocyte infiltration following BSCB disruption initiated inflammatory responses, leading to a secondary cascade of brain trauma due to the production of inflammatory mediators, such as TNF- $\alpha$, IL-1 $\beta$, IL-6 and iNOS (30). Therefore, we analyzed the effect of ETR blockade on the expression of inflammatory mediators by reverse-transcriptase PCR and ELISA assays at indicated time-points after SCI. Results showed that the mRNA expression levels of TNF- $\alpha$, IL-1 $\beta$ (at $2 \mathrm{~h}$ ), IL-6, and iNOS (at $6 \mathrm{~h}$ ) were upregulated post-injury, and blockade of ETAR and ETBR significantly reduced the levels of these inflammatory mediators compared with VEHs (Fig. 2A-D). In addition, the blockade of only ETAR, but not ETBR, decreased IL-1 $\beta$ and IL-6 expression in spinal cords post-injury (Fig. 2B and C). ELISA assays also showed that the blockade of the two ETRs significantly inhibited the production of TNF- $\alpha$, IL-1 $\beta$, IL-6 and iNOS at $24 \mathrm{~h}$ after SCI (Fig. 2E-H).

Reduction in oxidative stress in SCI mice following ETAR and ETBR blockade. Oxidative stress markers (HO-1, MDA and SOD) in injured mice with or without blockade of ETAR and ETBR were examined. SOD is an enzyme that neutralizes oxygen-free radicals and protects cells from being oxidized by superoxide toxicity (33). SOD is consumed during oxidative stress in a variety of pathological conditions. SCI caused a significant decrease in SOD level in SCI + VEH mice compared to SHAM mice, and the blockade of ETAR and ETBR significantly rescued the SOD level at $24 \mathrm{~h}$ after SCI (Fig. 3A). However, the blockade of only ETAR or ETBR did not rescue the SOD level in SCI mice (Fig. 3A). Similarly, 

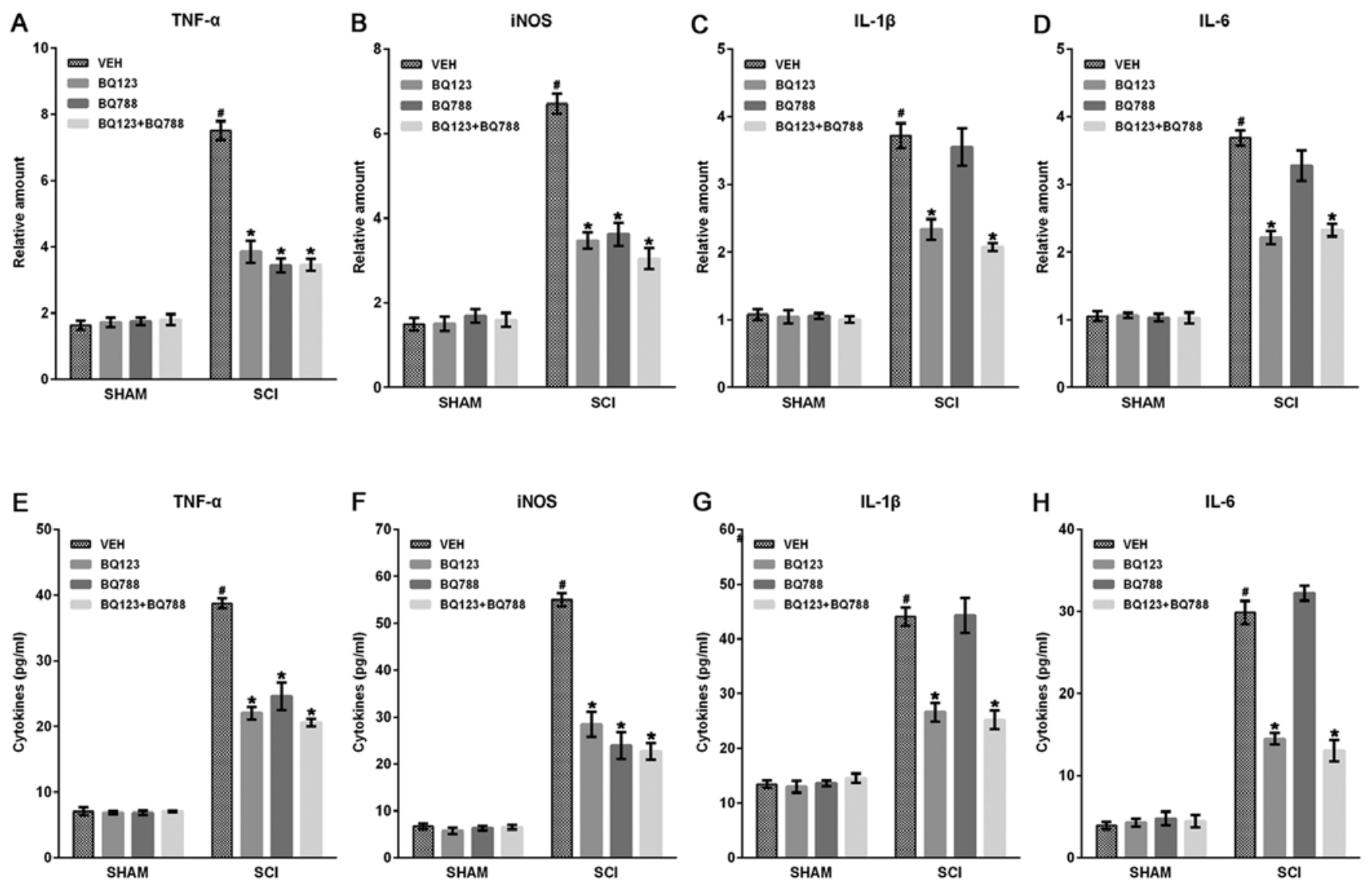

Figure 2. Treatment with BQ123 and/or BQ788 inhibits the expression of cytokines and inflammatory mediators in spinal cord injury (SCI) mice. Data represent mean \pm standard error of the mean (SEM) of three independent experiments. " $\mathrm{P}<0.05$, SCI mice treated with BQ123 and/or BQ788 vs. SCI + vehicle control (VEH) mice; ${ }^{\prime} \mathrm{P}<0.01, \mathrm{SCI}+\mathrm{VEH}$ mice vs. SHAM mice (n=3/group). (A-D) Quantitative analysis of reverse transcriptase PCR of tumor necrosis factor- $\alpha$ (TNF- $\alpha$ ), interleukin-1 $\beta$ (IL-1 $\beta$ ) (at $2 \mathrm{~h}$ ), IL-6, inducible nitric oxide synthase (iNOS) (at $6 \mathrm{~h}$ ) in SHAM and SCI mice left untreated or treated with BQ123 and/or BQ788 post-injury. (E-H) Enzyme-linked immunosorbent assay (ELISA) of TNF- $\alpha$, IL-1 $\beta$, IL-6 and iNOS at $24 \mathrm{~h}$ in SHAM and SCI mice left untreated or treated with BQ123 and/or BQ788 post-injury.

HO-1, the inducible form of HO, is an important defense mechanism against early oxidative stress $(5,34)$. Immunoblot assays revealed a greater increase in HO-1 levels at $24 \mathrm{~h}$ in SCI mice with blockade of ETBR or both ETAR and ETBR compared to SCI + VEH mice (Fig. 3B). By contrast, no differences were observed between SCI mice with only ETAR blockade and SCI + VEH mice (Fig. 3B). Nonetheless, levels of MDA, the final product of lipid peroxidation (33), were increased at $24 \mathrm{~h}$ after SCI and reduced in SCI mice with ETAR and ETBR blockade, whereas no statistically significant differences were found in SCI mice with blockade of only ETAR or ETBR (Fig. 3C).

Blockade of ETAR and ETBR reduced MMP-9 expression following SCI. MMP-9 plays a critical role in early neutrophil infiltration and long-term functional impairment after SCI (26). The immunoblot results revealed that MMP-9 was markedly increased at $24 \mathrm{~h}$ in SCI + VEH mice compared to control and was reduced in SCI mice with blockade of ETAR, ETBR, or a combination thereof (Fig. 4A).

The pro- and active forms of MMP-9 were evaluated in SHAM and SCI mice by gelatin zymography. The two forms were significantly reduced in SCI mice that were depleted of ETAR, ETBR, or both ETRs 24-h post-injury (Fig. 4B).
Blockade of ETAR and ETBR improved functional recovery after SCI. To determine the effects of ETR blockade on the long-term functional recovery after SCI, locomotor performance was evaluated in the BMS open field test, on a rotarod, and on a grid-walking task for 6 weeks. Hind-limb movements were abolished immediately after SCI as assessed using the BMS scale (31). Although all the groups gradual improved in locomotor functional recovery after 3 days, this recovery was markedly higher between 21 and 42 days after SCI in the group with blockade of ETAR and ETBR (Fig. 5A). Subsequent assessment of the ability of SCI mice to maintain position on a rotarod and walk across a grid revealed similar functional improvements in SCI mice with blockade of ETAR and ETBR, relative to their respective controls (Fig. 5B-C).

\section{Discussion}

ETR activation in the injured spinal cord triggers several pathologic responses, including leukocyte recruitment, superoxide generation, and BSCB breakdown, which exacerbate damage and impair neurological recovery (19-25). Although strategies to block ETRs in the injured spinal cord can promote locomotor function recovery, less is known about which ETR subtype influences neurological recovery 
A

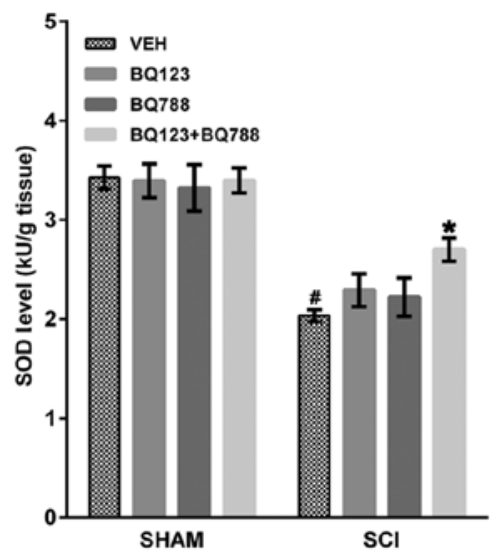

C

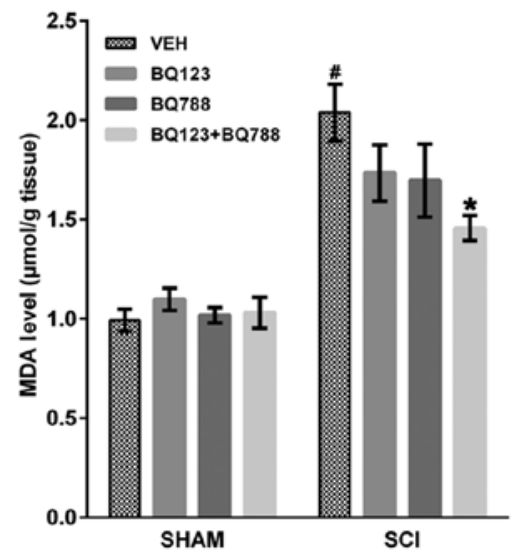

B
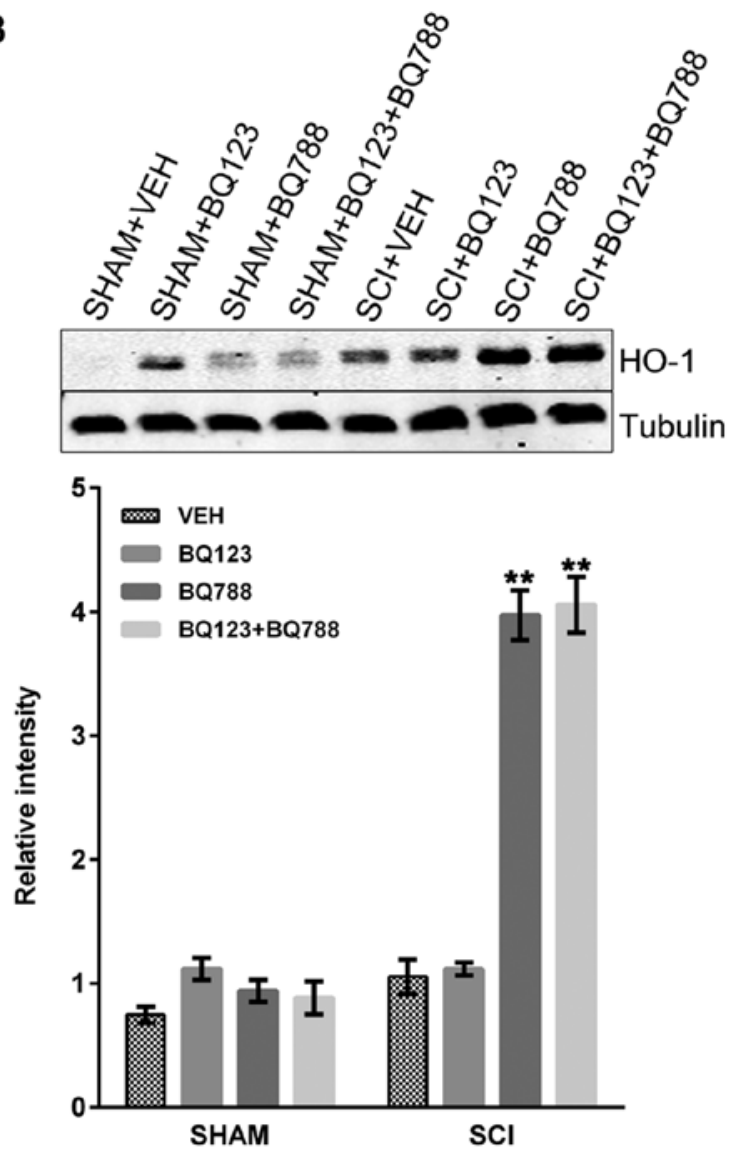

Figure 3. Treatment with BQ123 and/or BQ788 inhibits the expression of oxidative stress markers in spinal cord injury (SCI) mice. Data are presented as mean \pm standard error of the mean (SEM) of three independent experiments. ${ }^{*} \mathrm{P}<0.05,{ }^{* *} \mathrm{P}<0.01, \mathrm{SCI}$ mice treated with BQ123 and/or BQ788 vs. SCI + vehicle control (VEH) mice; ${ }^{\text {P }}<0.01$, SCI + VEH mice vs. SHAM mice ( $\mathrm{n}=3$ /group). (A) Enzyme-linked immunosorbent assay (ELISA) of SOD at $24 \mathrm{~h}$ in SHAM and SCI mice left untreated or treated with BQ123 and/or BQ788 post-injury. (B) Quantitative analysis of western blot of HO-1 at $24 \mathrm{~h}$ in SHAM and SCI mice left untreated or treated with BQ123 and/or BQ788 post-injury. Data are presented as mean \pm SEM of three independent experiments. (C) ELISA of MDA at $24 \mathrm{~h}$ in SHAM and SCI mice left untreated or treated with BQ123 and/or BQ788 post-injury. MDA, malondialdehyde.

processes after SCI $(18,35,36)$. In this study, we have shown that blockade of ETAR and ETBR in SCI mice resulted in an early reduction in leukocyte infiltration, oxidative stress, and expression of inflammatory mediators and MMP-9. Notably, although all the SCI mice with blockade of ETAR or ETBR showed an early improvement in locomotor function, blockade of the two receptor types resulted in significant long-term locomotor function improvement. Collectively, these results provide evidence for the additive cooperation between ETAR and ETBR in influencing early secondary pathogenesis and long-term locomotor recovery after SCI.

BQ123 is a selective ETAR antagonist that has been used as a biochemical tool to investigate ETR function (37), whereas BQ788 is a selective ETBR antagonist used to demonstrate the role of ET-1 and ETBR in physiological and pathophysiological conditions (29). Results of previous studies have indicated that BQ123 and BQ788 may exert anti-inflammatory and anti-oxidative properties in patients with ischemic heart disease $(27-29,38)$. In our experiments, the mice were treated with BQ123 and BQ788 pre- and post-SCI, which reduced ETAR and ETBR activity in the injured tissue, respectively. Thus, we assessed the specific roles of ETAR and ETBR in the injured spinal cord by using BQ123 and BQ788 in SCI mice.

Inflammatory neutrophils and monocytes are the first leukocytes to infiltrate the CNS after SCI (39-41). The number of circulating neutrophils increased $12-24$ h post-injury $(32,40)$, whereas the monocytes infiltrated into the CNS as early as day 1, reaching peak levels between 4 and 7 days after SCI (32). In our experiments, an increased number of circulating neutrophils and monocytes was detected in the peripheral blood in SCI mice. We also found that blockade of ETAR or ETBR pre- and post-SCI reduced circulating neutrophil numbers. Nevertheless, the blockade of ETBR or a combination of the two ETR subtypes decreased circulating monocytes in SCI mice. However, blockade of only ETAR had no effect on the number of monocytes after SCI. In parallel with this result, the expression levels of inflammatory mediators, such as TNF- $\alpha$, IL-1 $\beta$, IL-6 and iNOS, were upregulated after SCI, while 
A
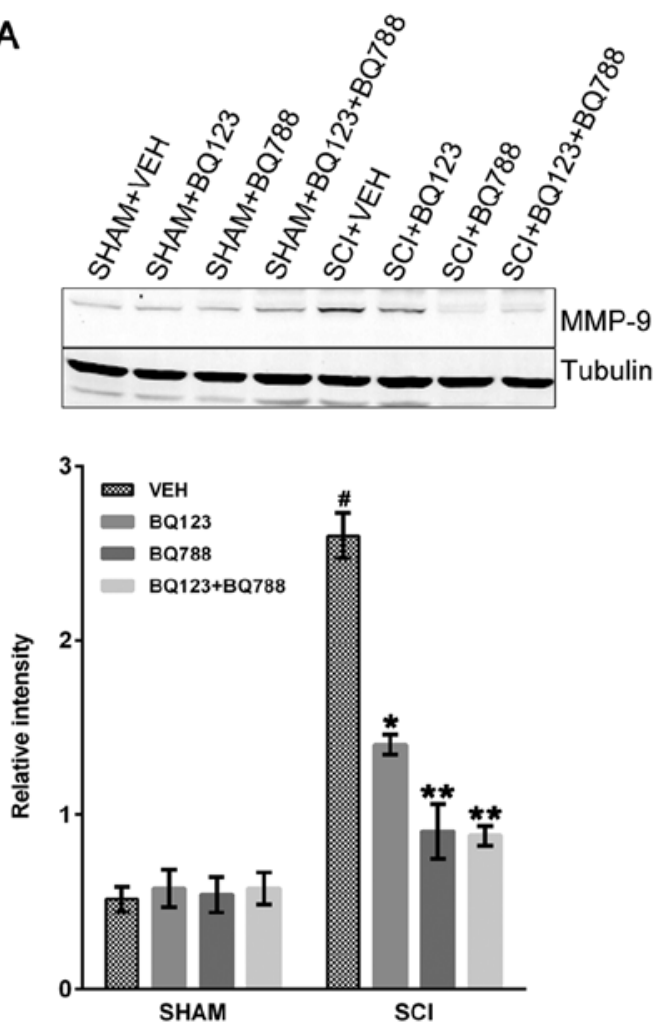

B
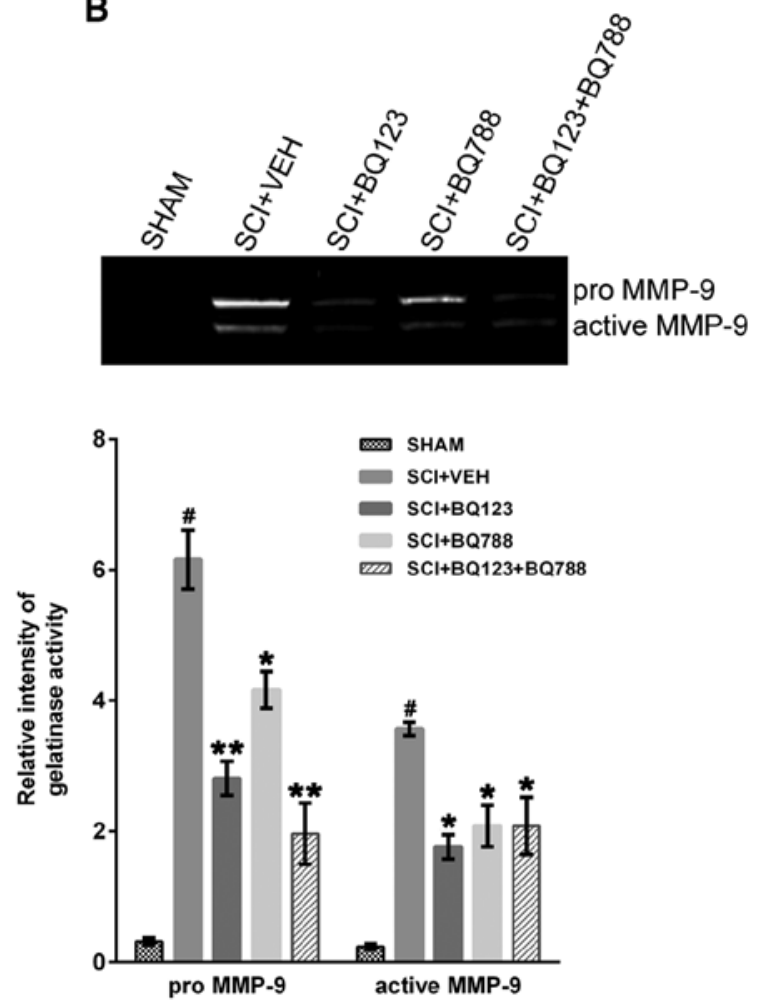

Figure 4. Treatment with BQ123 and/or BQ788 inhibits the expression of matrix metalloproteinase-9 (MMP-9) in spinal cord injury (SCI) mice. Data are the mean \pm standard error of the mean (SEM) of three independent experiments. ${ }^{*} \mathrm{P}<0.05,{ }^{* *} \mathrm{P}<0.01$, SCI mice treated with BQ123 and/or BQ788 vs. SCI + vehicle control (VEH) mice; ${ }^{\# P}<0.01$, SCI + VEH mice vs. SHAM mice (n=3/group). (A) Quantitative analysis of western blot of MMP-9 at 24 h in SHAM and SCI mice left untreated or treated with BQ123 and/or BQ788 post-injury. (B) Quantitative analysis of gelatin zymography of MMP-9 at 24 h in SHAM and SCI mice left untreated or treated with BQ123 and/or BQ788 post-injury.

the expression levels were significantly reduced following blockade of ETAR and ETBR. In addition, blockade of only ETBR did not decrease IL-1 $\beta$ and IL-6 expression levels in SCI mice. These results were consistent with previous findings (42-47) suggesting that the relationship between ETAR and ETBR in SCI mice is cooperative, and not antagonistic. The specific blockade of the two ETRs may inhibit inflammatory responses by suppressing the expression of inflammatory chemokines and reducing leukocyte infiltration following SCI-induced inflammatory mediator production.

Oxidative stress plays a critical role in secondary pathogenesis following SCI as it can lead to inflammation and apoptotic cell death. Increased oxidative stress reflects an imbalance between ROS and anti-oxidant levels $(17,32)$. We assessed three oxidative stress parameters in SCI mice: HO-1, MDA and SOD. MDA is a reactive aldehyde resulting from the degradation of polyunsaturated lipids that causes toxic stress in cells (7). However, endogenous anti-oxidative enzymes SOD and HO-1 catalyze superoxide into oxygen and hydrogen peroxide $(7,8,33,48)$. Consistent with previous observations $(7,8,33,48)$, SCI induced an increase in MDA and reduced HO-1 and SOD expression. To the best of our knowledge, the results have shown for the first time that blockade of ETAR and ETBR in SCI mice significantly reversed reduction in MDA and SOD levels, thereby restoring the oxidative stress balance.

HO-1 is regarded as a sensitive and reliable indicator of cellular oxidative stress $(7,8)$. Increased HO-1 expression in SCI rodent models can reduce neural tissue damage and improve locomotor function $(32,49)$. HO-1 induction is regulated by the stress-responsive element and the Maf recognition element. Furthermore, HO-1 is rapidly induced by the specific regulation of oxidant-responsive transcription factors AP-1, $\mathrm{NF}-\kappa \mathrm{B}$, and Nrf2/Keap1-Bach1 in oxidative stress $(49,50)$. We found that blockade of ETBR or the two ETR subtypes resulted in a significant elevation of HO-1 expression in SCI mice, whereas the blockade of only ETAR had no effect. These results may be due to the critical role of ETBR on the ERK pathway signaling, which may affect the Nrf2/Keap1-Bach1 equilibrium, resulting in a decreased HO-1 expression $(50,51)$.

Pro- and active MMP-9 is markedly accumulated in acute $\mathrm{SCI}$, and it is able to degrade components of the extracellular matrix, such as microvasculature basal lamina and myelin basic protein $(26,32)$. MMP-9 is derived primarily from neutrophils, although it can also originate from monocytes. It is likely that MMP-9 is released in response to activation of these cells following SCI. MMP-9 has been shown to contribute to early BSCB disruption, leukocyte infiltration, and white matter damage in SCI rodents (26,32). Furthermore, MMP-9-deficient mice show functional improvement in locomotion following cerebral focal ischemia (52). Similar to previous studies (30), we detected an increased MMP-9 expression and activity in SCI mice. We found that blockade of ETAR, ETBR, or a combination thereof significantly reduced levels of pro- and active forms of MMP-9. These results are highly dependent 


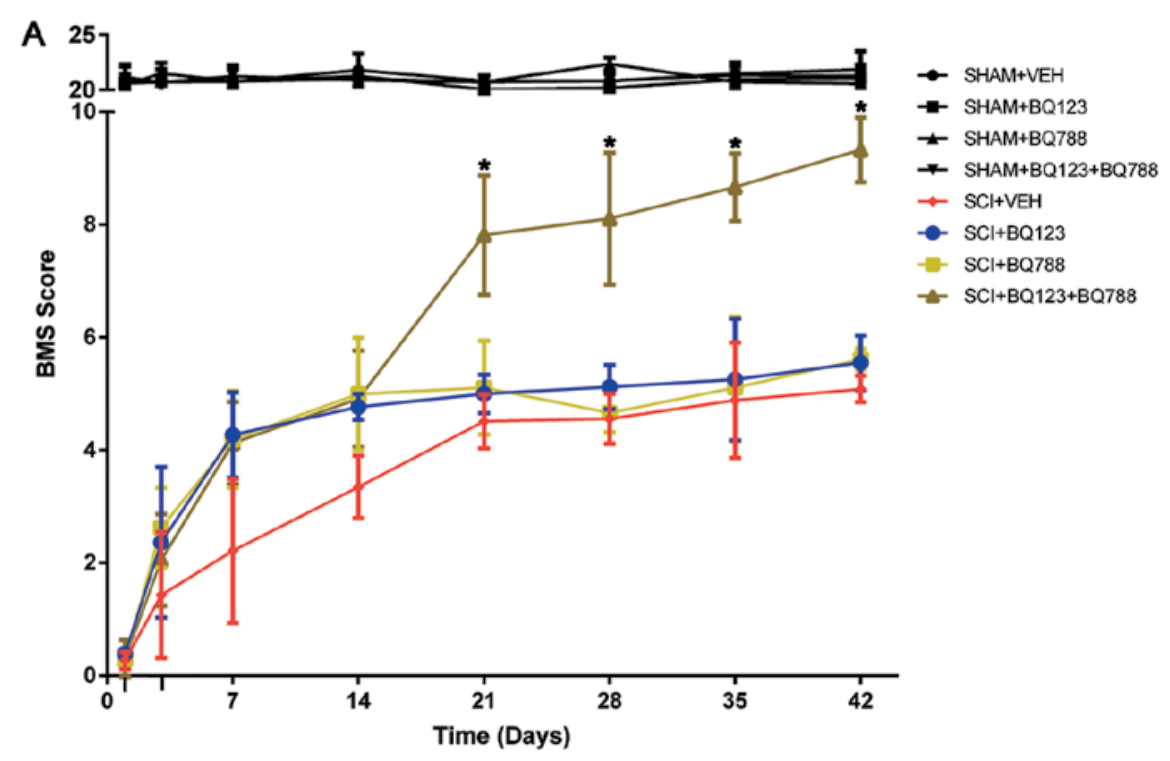

B

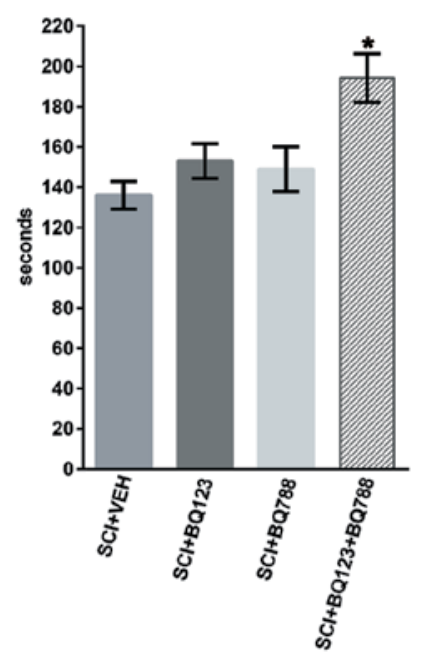

C

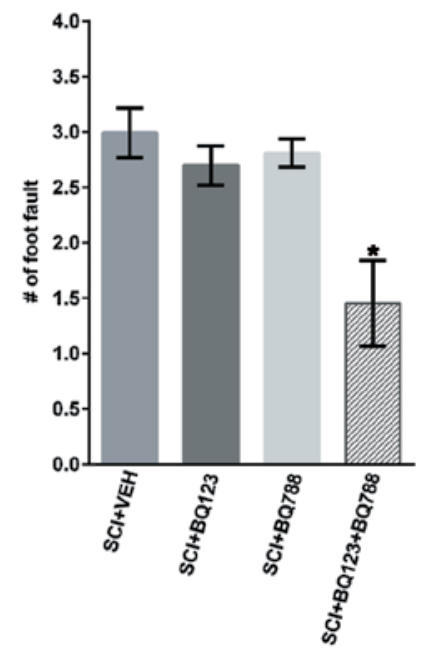

Figure 5. Treatment with BQ123 and/or BQ788 improves long-term functional recovery after spinal cord injury (SCI). Data are the mean \pm standard error of the mean (SEM) of three independent experiments. "P<0.05, SCI + BQ123 + BQ788 mice vs. SCI + vehicle control (VEH) mice (n=10/group). (A) Functional recovery as determined by the Basso Mouse Scale (BMS) in mice treated with BQ123 and/or BQ788. (B) Performance on a rotarod in BQ123- and/or BQ788-treated SCI mice. (C) Comparison of grid walking in BQ123 and/or BQ788 treated SCI mice.

on the blockade of ETRs, which reduces leukocyte infiltration after SCI.

It was previously reported that use of pharmacological or genetic strategies to block ET signaling enhanced the recovery of locomotor function in SCI rodents $(23,25)$. In our experiments, although blockade of only ETAR or ETBR in SCI mice gradually enhanced locomotor function recovery, improved long-term locomotor recovery was limited to SCI mice with blockade of ETAR and ETBR. These beneficial effects may be partially due to improvement of the SCI molecular environment, including reductions in inflammation, oxidative stress, and MMP-9 activation, all of which are dependent on cooperativity between the two ETR subtypes $(26,32,48)$. It is also possible that ETAR or ETBR exert distinct effects on pro-inflammatory and oxidative stress states in SCI, which may subsequently influence neurological functional recovery. For example, blockade of vascular ETAR and ETBR and astrocyte ETBR after SCI may reduce ischemia and astrogliosis and facilitate neuronal survival, regeneration, and neurological function recovery (23). Further studies are needed to address the involvement of specific ETR subtypes in SCI.

Taken together, to the best of our knowledge, findings of this study provide the first evidence for collaboration between ETAR and ETBR in creating an environment that is hostile to neurological recovery in SCI. These results may serve as a foundation for developing combination ET-based therapy against multiple targets that alleviate early inflammatory response and oxidative stress and promote long-term functional recovery following SCI. Although the mechanisms involved in ETR blockade protection against SCI in mice requires further investigation, our data suggest that ETR blockade is an 
effective treatment for SCI and may be used to improve SCI recovery in humans in the future.

\section{Acknowledgements}

This study was supported by the Science and Technology Bureau Foundation of Ningbo (no. 2012A610230).

\section{References}

1. Hawkins B and Davis T: The blood-brain barrier/neurovascular unit in health and disease. Pharmacol Rev 57: 173-185, 2005.

2. Abbott N, Rönnbäck L and Hansson E: Astrocyte-endothelial interactions at the blood-brain barrier. Nat Rev Neurosci 7: 41-53, 2006.

3. Bao F, Chen Y, Schneider K and Weaver L: An integrin inhibiting molecule decreases oxidative damage and improves neurological function after spinal cord injury. Exp Neurol 214: 160-167, 2008

4. Goldbaum $\mathrm{O}$ and Richter-Landsberg C: Stress proteins in oligodendrocytes: differential effects of heat shock and oxidative stress. J Neurochem 78: 1233-1242, 2001.

5. Lin Q, Weis S, Yang G, et al: Heme oxygenase-1 protein localizes to the nucleus and activates transcription factors important in oxidative stress. J Biol Chem 282: 20621-20633, 2007.

6. Lindsey M, Wedin K, Brown M, et al: Matrix-dependent mechanism of neutrophil-mediated release and activation of matrix metalloproteinase 9 in myocardial ischernia/reperfusion. Circulation 103: 2181-2187, 2001.

7. Liu D, Li L and Augustus L: Prostaglandin release by spinal cord injury mediates production of hydroxyl radical, malondialdehyde and cell death: a site of the neuroprotective action of methylprednisolone. J Neurochem 77: 1036-1047, 2001.

8. Liu Y, Tachibana T, Dai Y, et al: Heme oxygenase-1 expression after spinal cord injury: the induction in activated neutrophils. J Neurotrauma 19: 479-490, 2002.

9. Nguyen H, O'Barr T and Anderson A: Polymorphonuclear leukocytes promote neurotoxicity through release of matrix metalloproteinases, reactive oxygen species, and TNF-alpha. J Neurochem 102: 900-912, 2007.

10. Dang A, Tay B, Kim H, Nauth A, Alfonso-Jaume M and Lovett D: Inhibition of MMP2/MMP9 after spinal cord trauma reduces apoptosis. Spine (Phila Pa 1976) 33: E576-E579, 2008.

11. Dehouck M, Vigne P, Torpier G, Breittmayer J, Cecchelli R and Frelin C: Endothelin-1 as a mediator of endothelial cell-pericyte interactions in bovine brain capillaries. J Cereb Blood Flow Metab 17: 464-469, 1997.

12. Hama H, Kasuya Y, Sakurai T, et al: Role of endothelin-1 in astrocyte responses after acute brain damage. J Neurosci Res 47: 590-602, 1997

13. Siren A, Knerlich F, Schilling L, Kamrowski-Kruck H, Hahn A and Ehrenreich H: Differential glial and vascular expression of endothelins and their receptors in rat brain after neurotrauma. Neurochem Res 25: 957-969, 2000.

14. Martin D, Schoenen J, Delrée P, et al: Experimental acute traumatic injury of the adult rat spinal cord by a subdura inflatable balloon: methodology, behavioral analysis, and histopathology. J Neurosci Res 32: 539-550, 1992.

15. Lampl Y, Fleminger G, Gilad R, Galron R, Sarova-Pinhas I and Sokolovsky M: Endothelin in cerebrospinal fluid and plasma of patients in the early stage of ischemic stroke. Stroke 28: 1951-1955, 1997

16. Reijerkerk A, Lakeman K, Drexhage J, et al: Brain endothelial barrier passage by monocytes is controlled by the endothelin system. J Neurochem 121: 730-737, 2012.

17. Leonard M, Briyal S and Gulati A: Endothelin B receptor agonist, IRL-1620, reduces neurological damage following permanent middle cerebral artery occlusion in rats. Brain Res 1420: 48-58, 2011.

18. Chou A, Chen T, Winardi W, et al: Functional neuroprotective effect of CGS 26303, a dual ECE inhibitor, on ischemic-reperfusion spinal cord injury in rats. Exp Biol Med (Maywood) 232. 214-218, 2007.

19. Barnes K and Turner A: The endothelin system and endothelinconverting enzyme in the brain: molecular and cellular studies. Neurochem Res 22: 1033-1040, 1997.
20. Kallakuri S, Kreipke C, Schafer P, Schafer S and Rafols J: Brain cellular localization of endothelin receptors $\mathrm{A}$ and $\mathrm{B}$ in a rodent model of diffuse traumatic brain injury. Neuroscience 168: 820-830, 2010.

21. McKenzie A, Hall J, Aihara N, Fukuda K and Noble L: Immunolocalization of endothelin in the traumatized spinal cord: relationship to blood-spinal cord barrier breakdown. J Neurotrauma 12: 257-268, 1995.

22. MacCumber M, Ross C and Snyder S: Endothelin in brain: receptors, mitogenesis, and biosynthesis in glial cells. Proc Natl Acad Sci USA 87: 2359-2363, 1990.

23. Peters C, Rogers S, Pomonis J, et al: Endothelin receptor expression in the normal and injured spinal cord: potential involvement in injury-induced ischemia and gliosis. Exp Neurol 180: 1-13, 2003.

24. Huggins J, Pelton J and Miller R: The structure and specificity of endothelin receptors: their importance in physiology and medicine. Pharmacol Ther 59: 55-123, 1993.

25. Koyama Y, Takemura M, Fujiki K, Ishikawa N, Shigenaga $Y$ and Baba A: BQ788, an endothelin ET(B) receptor antagonist, attenuates stab wound injury-induced reactive astrocytes in rat brain. Glia 26: 268-271, 1999.

26. Noble L, Donovan F, Igarashi T, Goussev S and Werb Z: Matrix metalloproteinases limit functional recovery after spinal cord injury by modulation of early vascular events. J Neurosci 22 : 7526-7535, 2002.

27. Fu L, Guo Z and Longhurst J: Endogenous endothelin stimulates cardiac sympathetic afferents during ischaemia. J Physiol 588: 2473-2486, 2010

28. Douglas SA, Vickery-Clark LM, Louden C and Ohlstein EH: Selective ETA receptor antagonism with BQ-123 is insufficient to inhibit angioplasty induced neointima formation in the rat. Cardiovasc Res 29: 641-646, 1995.

29. Okada M and Nishikibe M: BQ-788, a selective endothelin ET(B) receptor antagonist. Cardiovasc Drug Rev 20: 53-66, 2002.

30. Lee J, Kim H, Choi H, Oh T and Yune T: Fluoxetine inhibits matrix metalloprotease activation and prevents disruption of blood-spinal cord barrier after spinal cord injury. Brain 135: 2375-2389, 2012

31. Basso D, Fisher L, Anderson A, Jakeman L, McTigue D and Popovich P: Basso mouse scale for locomotion detects differences in recovery after spinal cord injury in five common mouse strains. J Neurotrauma 23: 635-659, 2006.

32. Lee S, Rosen S, Weinstein P, van Rooijen N and Noble-Haeusslein L: Prevention of both neutrophil and monocyte recruitment promotes recovery after spinal cord injury. J Neurotrauma 28: 1893-1907, 2011.

33. Xu W, Chi L, Xu R, et al: Increased production of reactive oxygen species contributes to motor neuron death in a compression mouse model of spinal cord injury. Spinal Cord 43: 204-213, 2005.

34. Cuadrado A and Rojo A: Heme oxygenase-1 as a therapeutic target in neurodegenerative diseases and brain infections. Curr Pharm Des 14: 429-442, 2008.

35. Salzman S, Acosta R, Beck G, Madden J, Boxer B and Ohlstein E: Spinal endothelin content is elevated after moderate local trauma in the rat to levels associated with locomotor dysfunction after intrathecal injection. J Neurotrauma 13: 93-101, 1996.

36. Nagasaka J, Tsuji M, Takeda $\mathrm{H}$ and Matsumiya T: Role of endothelin receptor subtypes in the behavioral effects of the intracerebroventricular administration of endothelin-1 in conscious rats. Pharmacol Biochem Behav 64: 171-176, 1999.

37. Ishikawa K, Fukami T, Nagase T, et al: Cyclic pentapeptide endothelin antagonists with high ETA selectivity. Potency- and solubility-enhancing modifications. J Med Chem 35: 2139-2142, 1992.

38. Khimji A and Rockey D: Endothelin - biology and disease. Cell Signal 22: 1615-1625, 2010.

39. Beck K, Nguyen H, Galvan M, Salazar D, Woodruff T and Anderson A: Quantitative analysis of cellular inflammation after traumatic spinal cord injury: evidence for a multiphasic inflammatory response in the acute to chronic environment. Brain 133: 433-447, 2010

40. Letellier E, Kumar S, Sancho-Martinez I, et al: CD95-ligand on peripheral myeloid cells activates Syk kinase to trigger their recruitment to the inflammatory site. Immunity 32: 240-252, 2010.

41. Stirling D and Yong V: Dynamics of the inflammatory response after murine spinal cord injury revealed by flow cytometry. J Neurosci Res 86: 1944-1958, 2008. 
42. Ahnstedt H, Stenman E, Cao L, Henriksson M and Edvinsson L: Cytokines and growth factors modify the upregulation of contractile endothelin ET(A) and ET(B) receptors in rat cerebral arteries after organ culture. Acta Physiol (Oxf) 205: 266-278, 2012.

43. Zarpelon A, Pinto L, Cunha T, et al: Endothelin-1 induces neutrophil recruitment in adaptive inflammation via $\mathrm{TNF} \alpha$ and CXCL1/CXCR2 in mice. Canadian Can J Physiol Pharmacol 90: 187-199, 2012

44. Tonari M, Kurimoto T, Horie T, Sugiyama T, Ikeda T and Oku H: Blocking endothelin-B receptors rescues retinal ganglion cells from optic nerve injury through suppression of neuroinflammation. Invest Ophthalmol Vis Sci 53: 3490-3500, 2012.

45. Wang $\mathrm{H}$, Hsieh $\mathrm{H}$ and Yang $\mathrm{C}$ : Nitric oxide production by endothelin-1 enhances astrocytic migration via the tyrosine nitration of matrix metalloproteinase-9. J Cell Physiol 226: 2244-2256, 2011.

46. Gallelli L, Pelaia G, D'Agostino B, et al: Endothelin-1 induces proliferation of human lung fibroblasts and IL-11 secretion through an ET(A) receptor-dependent activation of MAP kinases. J Cell Biochem 96: 858-868, 2005.

47. Solini A, Santini E, Madec S, Cuccato S and Ferrannini E: Effects of endothelin-1 on fibroblasts from type 2 diabetic patients: possible role in wound healing and tissue repair. Growth Factors 25: 392-399, 2007.
48. Yin X, Yin Y, Cao F, et al: Tanshinone IIA attenuates the inflammatory response and apoptosis after traumatic injury of the spinal cord in adult rats. PLoS One 7: e38381, 2012.

49. Kanno H, Ozawa H, Dohi Y, Sekiguchi A, Igarashi K and Itoi E: Genetic ablation of transcription repressor Bach1 reduces neural tissue damage and improves locomotor function after spinal cord injury in mice. J Neurotrauma 26: 31-39, 2009.

50. Goven D, Boutten A, Lecon-Malas V, Boczkowski J and Bonay M: Prolonged cigarette smoke exposure decreases heme oxygenase- 1 and alters Nrf2 and Bach1 expression in human macrophages: roles of the MAP kinases ERK(1/2) and JNK. FEBS Lett 583: 3508-3518, 2009.

51. Sallum CO, Wilson JL, Rupasinghe C, et al: Enhancing and limiting endothelin-1 signaling with a cell-penetrating peptide mimicking the third intracellular loop of the ETB receptor. Chem Biol Drug Des 80: 374-381, 2012.

52. Romanic AM1, White RF, Arleth AJ, Ohlstein EH and Barone FC: Matrix metalloproteinase expression increases after cerebral focal ischemia in rats: inhibition of matrix metalloproteinase-9 reduces infarct size. Stroke 29: 1020-1030, 1998. 